\title{
Tunable polaritons of spiral nanowire metamaterials
}

\author{
Thanos IOANNIDIS ${ }^{1}$, TATJANA GRIC ${ }^{1,2,3^{*}}$, EdIK RAFAILOV 2,4 \\ ${ }^{1}$ Department of Electronic Systems, Vilnius Gediminas Technical University, Vilnius, Lithuania \\ ${ }^{2}$ Aston Institute of Photonic Technologies, Aston University, Birmingham B4 7ET, UK \\ ${ }^{3}$ Semiconductor Physics Institute, Center for Physical Sciences and Technology, Vilnius, Lithuania \\ ${ }^{4}$ Peter the Great St. Petersburg Polytechnic University, St. Petersburg, Russia \\ *tatjana.gric@vgtu.It
}

\begin{abstract}
The tunable spiral nanowire metamaterial design at optical frequency is presented, and the surface polaritons are theoretically studied. It was found that the dispersions of the polaritons could be tuned by varying physical dimensions of the spiral nanowire metamaterial. This geometry is unique. Doing so, one may dynamically control the properties of surface polaritons. In addition, the Ferrell-Berreman modes can be excited that is impossible with the regular nanowire metamaterials having the circular cross-section of the nanowires. Herein, the presence of Ferrel-Berreman branches is confirmed by the performed analysis of the metamaterial band structure. It is worthwhile noting, that existence of FerrelBerreman modes is possible without epsilon-near-zero (ENZ) regime. The design of devices where Ferrell-Berreman modes can be exploited for practical applications ranging from plasmonic sensing to imaging and absorption enhancement is possible because of the propagation constant revealing subtle microscopic resonances.
\end{abstract}

\section{Introduction}

Surface plasmons (SPs) are known as the collective oscillations of the delocalized electrons existing at metal-dielectric interfaces. Owing to their ability to confine light in subwavelength dimensions with high efficiency, SPs offer a route to overcome the diffraction limit of classical optics $[1,2]$. This enables a wide range of applications including surface enhanced spectroscopy $[3,4]$, biomedical sensing [5, 6], solar cell photovoltaics [7, 8], and optical antennas $[9,10]$ since surface-plasmon-based circuits are known to merge the fields of photonics and electronics at the nanoscale, thereby enabling them to overcome the existing difficulties related to the large size mismatch between the micrometer-scale bulky components of photonics and the nanometer-scale electronic chips. These applications, however, are generally limited to high electromagnetic (EM) frequencies (the UV, visible, and near-infrared ranges). This is because metals behave akin to perfect electric conductors (PECs) at lower frequency regimes and do not support SP modes. Early works showed that by corrugating metal surfaces, this limitation can be overcome, and they reported the excitation of highly confined SP-like electromagnetic modes at microwave frequencies [11, 12]. In 2004, Pendry et al. introduced the concept of spoof surface plasmons (spoof SPs) and demonstrated that plasmonic metamaterials constructed by patterning metal surfaces with subwavelength periodic features can mimic, at low frequencies (far IR, terahertz, or microwave regimes), the EM guiding characteristics of optical SPs [13, 14]. It should be mentioned that the exceptional properties of SPPs, such as, subwavelength confinement and strong field enhancement give rise to a wide range of innovative applications [15-17].

Since most of the conventional nanowire metamaterials can be controlled by the limited number of geometrical parameters, the focus of research has been moved to the possibility of tunable SPs by dealing with spiral nanowire matamaterial. Herein, the ability of spiral nanowire metamaterials to support unique absorption resonances related to radiative bulk plasmon-polaritons is theoretically demonstrated. The properties of these radiative bright modes significantly differ from the ones of the conventional dark modes (surface plasmon 
polaritons). The unique absorption resonances manifested in our metamaterials were originally studied by Ferrell in 1958 for plasmon-polaritonic thin-films in the ultraviolet [18], and by Berreman in 1963 for phonon-polaritonic thin-films in the mid-infrared spectral region [19]. One key for reaching the broadened SPs existence region is the tunability of the spiral angle.

In this work, we develop an effective medium theoretical model for the analytical description of spiral-shaped spoof devices and use it to investigate quantitatively their spectral properties. This model allows for a comprehensive understanding of FB modes supported by the structure.

\section{Theoretical model}

Herein, we focus on a 2D wire whose surface is decorated by $N$ spiral-shaped grooves filled with a dielectric material of refractive index $n_{g}$. The resulting inner and outer radii, which correspond to the bottom and opening of the grooves, are $r$ and $R$, respectively, as shown in Fig. 1. The period and width of the grooves along the wire perimeter are $d$ and $a$. The spiral is built in such a way that the intersection angle between the tangent to each spiral arm and the radial direction is the same along the spiral length; see Fig. 1. These are the so-called logarithmic spirals, characterized by a spiral angle $\theta$, and can be parametrized as

$$
\begin{aligned}
& x(t)=r e^{t / \cos \theta} \cos t \\
& y(t)=r e^{t / \cos \theta} \sin t
\end{aligned}
$$

In the limit $a<d \square \lambda$, a metamaterial approximation, in which the textured PEC is treated a homogeneous effective medium, can be applied. In this homogeneous metamaterial picture, the effective permittivity and permeability in the region between the inner and outer radii acquire a diagonal, spatially independent tensor form. The component of the permittivity normal to the spiral arms (z-direction) is given by $\varepsilon_{n}=n_{g}^{2} d / a$, while the parallel components

diverge. The permeability components are set so that the EM radiation propagates at the speed of light inside the spiral grooves. This procedure yields the following effective permittivity and permeability tensors for TM waves expressed in cylindrical coordinates:

$$
\begin{aligned}
& \overline{\bar{\varepsilon}}^{-1}=\frac{a}{2 n_{g}^{2} d}\left[\begin{array}{cc}
1-\cos 2 \theta & -\sin 2 \theta \\
-\sin 2 \theta & 1+\cos 2 \theta
\end{array}\right] \\
& \mu_{z}^{-1}=\frac{d}{a}
\end{aligned}
$$

where the permittivity tensor is restricted to the $x y$-plane.

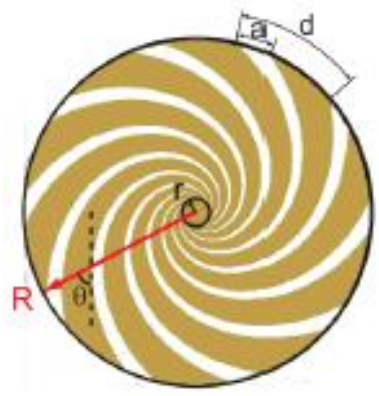


Fig. 1. Localized spoof surface plasmons in a 2D subwavelength PEC wire corrugated with spiral grooves. Cross-section of the corrugated PEC wire with the inner and outer radii $r$ and $R$, periodicity $d$, groove width $a$, and the spiral angle $\theta$.

\section{Modelling and the analytical solution}

The proposed geometry of the nanowire composites is shown in Fig. 2. Nanowires with permittivity $\varepsilon_{m}^{M}$ are embedded in a dielectric host material with permittivity $\varepsilon_{d}^{M}=2.4$.

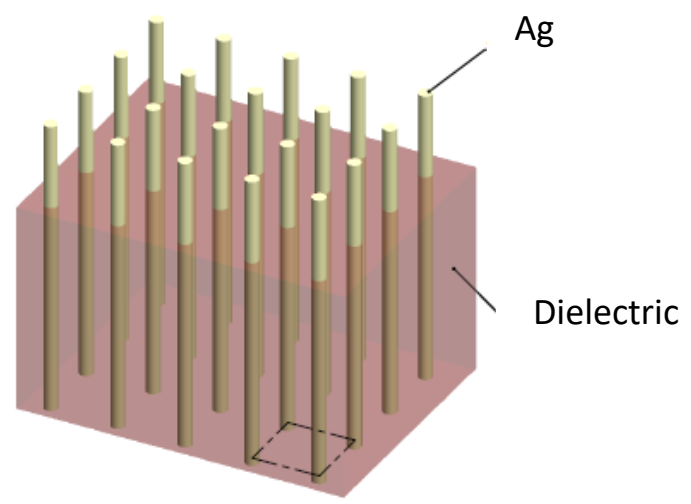

Fig. 2. Schematic view of a nanowire composite.

On the basis of an effective medium approximation we evaluate the effective permittivities of the nanowire metamaterial according to:

$$
\begin{aligned}
& \varepsilon_{\perp}^{M}=\varepsilon_{d}^{M}\left[\frac{\varepsilon_{m}^{M}\left(1+\rho^{M}\right)+\varepsilon_{d}^{M}\left(1-\rho^{M}\right)}{\varepsilon_{m}^{M}\left(1-\rho^{M}\right)+\varepsilon_{d}^{M}\left(1+\rho^{M}\right)}\right] \\
& \varepsilon_{\|}^{M}=\varepsilon_{m}^{M} \rho^{M}+\varepsilon_{d}^{M}\left(1-\rho^{M}\right)
\end{aligned}
$$

Here, subindex $\mathrm{M}$ refers to the metamaterial medium, and $\rho^{M}$ is the metal filling fraction ratio which is defined as:

$$
\rho^{M}=\frac{\text { nanowire area }}{\text { unitcell area }}
$$

To explore and demonstrate the properties of surface waves we adopt a Drude model to characterize the metal (i. e. silver), expressing the permittivity as $\varepsilon_{m}^{M}(\omega)=\varepsilon_{\infty}-\frac{\omega_{p}^{2}}{\omega^{2}+i \delta \omega}$. The parameters are obtained by fitting this permittivity function to a particular frequency range of bulk material [20]. It is found [21] that for silver, the values of $\varepsilon_{\infty}=5$, $\omega_{p}=2.2971 \cdot 10^{15} \mathrm{~Hz}, \delta=2.3866 \cdot 10^{13} \mathrm{~Hz}$ give a reasonable fit. We calculate the metal filling fraction $\left(\rho^{M}\right)$ based on the values of the pore diameter $\left(d^{M}\right)$ and spacing $\left(S^{M}\right)$ and, assuming a perfect rectangular structure, we apply the following equation [22]: 


$$
\rho^{M}=\frac{\pi\left(d^{M}\right)^{2}}{4\left(S^{M}\right)^{2}}
$$

With this assumption it is possible to derive a dispersion relation for the surface modes localized at the interface between metamaterial and $\mathrm{PbS}$. Evaluating the tangential components of the electric and magnetic fields at the interface it is then, in turn, possible to obtain a single surface mode with the propagation constant [23]

$$
\beta=k\left(\frac{\left(\varepsilon_{P b S}-\varepsilon_{\|}^{M}\right) \varepsilon_{P b S} \varepsilon_{\perp}^{M}}{\varepsilon_{P b S}^{2}-\varepsilon_{\perp}^{M} \varepsilon_{\|}^{M}}\right)^{1 / 2},
$$

where $k$ is the wavenumber (absolute value of the wavevector in vacuum) and $\beta$ is the component of the wavevector parallel to the interface.

By substituting effective permittivity in different direction from Eq. (3) in Eqs. (5), (6) we arrive at the effective permittivities of the spiral nanowire metamaterial as follows:

$$
\begin{aligned}
& \varepsilon_{\perp}^{S M}=-\varepsilon_{d}^{M} \frac{\varepsilon_{d}^{M}\left(\rho^{M}-1\right)+\frac{a d \varepsilon_{m}^{M} \sin (2 \theta)(\rho+1)}{2}}{\varepsilon_{d}^{M}\left(\rho^{M}+1\right)+\frac{a d \varepsilon_{m}^{M} \sin (2 \theta)(\rho-1)}{2}} \\
& \varepsilon_{\|}^{S M}=-\varepsilon_{d}^{M}\left(\rho^{M}-1\right)-\frac{a d \varepsilon_{m}^{M} \rho^{M}(\cos (2 \theta)+1)}{2}
\end{aligned}
$$

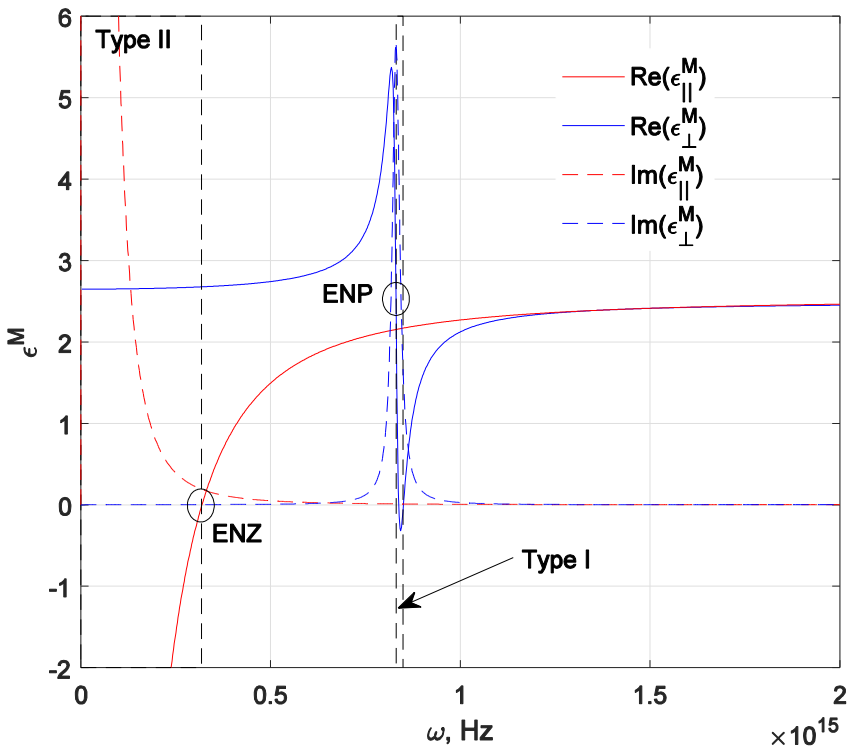

(a) 


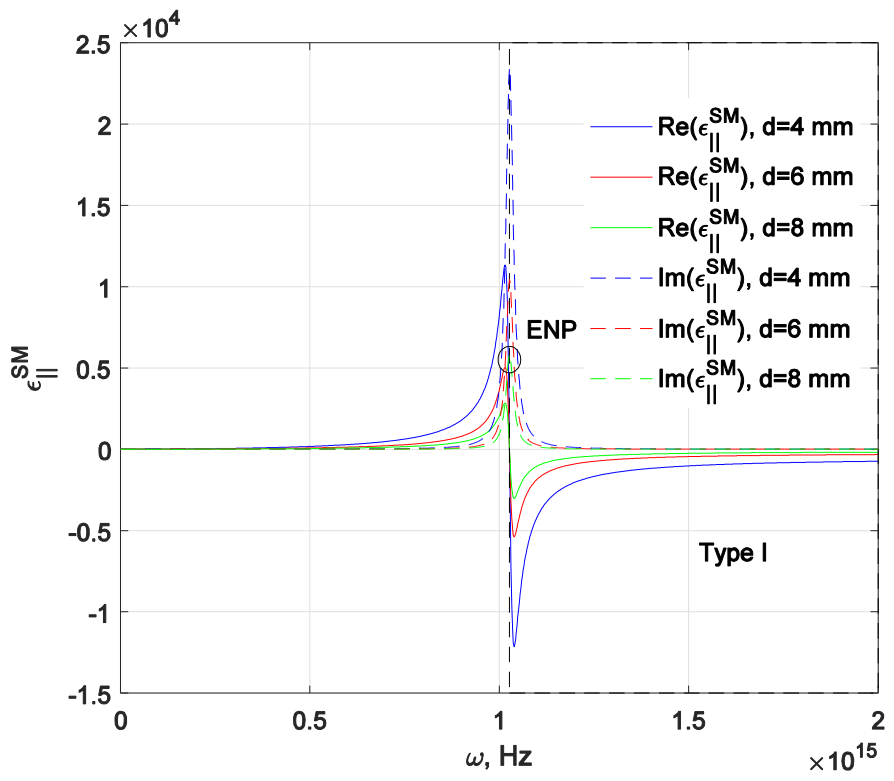

(b)

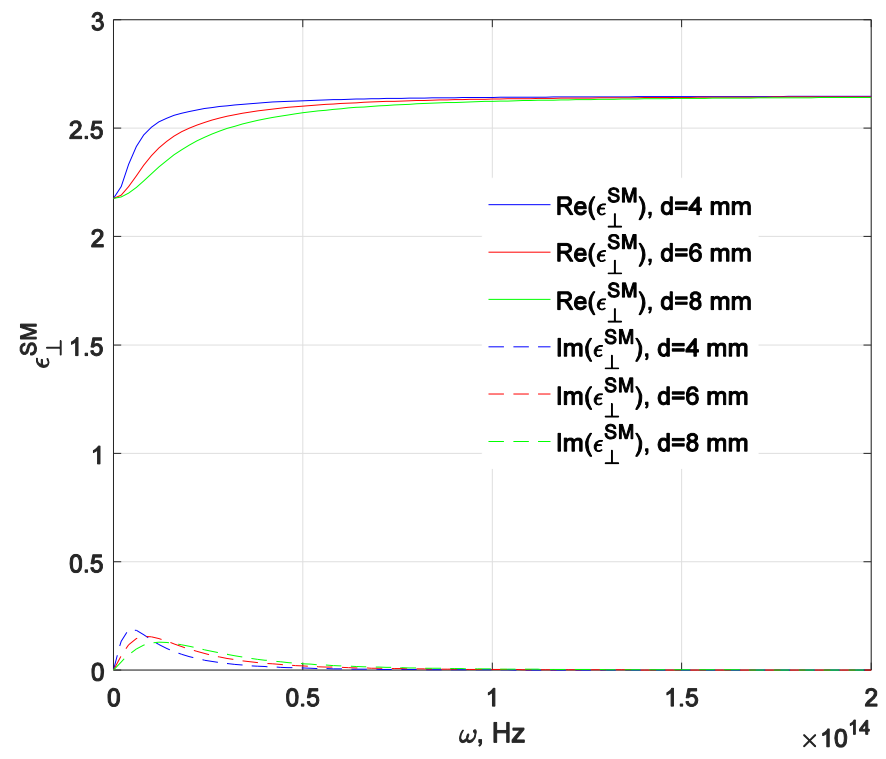

(c)

Fig. 3. (a) Nanowire system: Real part of the dielectric permittivity for a nanowire structure. (b), (c) Spiral nanowire system: Real part of the dielectric permittivity for a spiral nanowire structure.

In figure 3, we plot the effective medium constants for the spiral nanowire and regular nanowire structures using the homogenization formulae. The nanowire metamaterial structure shows an epsilon-near-zero effect as well as epsilon-near-pole resonance. Only the real parts are shown for clarity and the imaginary parts can be calculated similarly. The nanowire metamaterial structure consisting of metallic inclusions shows both Type I and Type II hyperbolic behavior. The spiral nanowire effective medium theory parameters are shown in 
figures 3 (b), (c). Type II behavior, which is difficult to achieve with spiral nanowire metamaterial structures, is observed. It also shows the characteristic epsilon-near-pole (ENP) resonance.

The most important aspect to note about the epsilon-near-zero (ENZ) and ENP resonances are the directions in which they occur for spiral nanowire and regular nanowire samples. The former issue dramatically changes the reflection and transmission spectrum of the two types of hyperbolic media. For the nanowire design, ENZ occurs along the nanowire length. This is intuitively expected since the Drude plasma frequency which determines the ENZ always occurs in the direction of free electron motion. On the contrary, the resonant ENP behavior of the two geometries occurs in the direction for which there is no continuous free electron motion. The ENP resonance occurs perpendicular to the wires in the nanowire geometry [24]. By presenting a modal analysis, we now show that the physical origin of the bulk absorption in metamaterials is due to the excitation of leaky bulk polaritons called Ferrel-Berreman modes [25-27]. Actually, the bulk metal forms volume charge oscillations at the ENZ of the metal (bulk or volume plasmons). It is worthwhile noting that these excitations are in the form of a completely longitudinal wave. Doing so, they cannot be excited with free space light (which a transverse wave). In case of films of metal with thicknesses less than the metal skin depth, the top and bottom interface couple. The former issue allows for collective charge oscillations across the film. Thus, the bulk plasmon is no longer purely longitudinal and interaction with free space light at frequencies near the metal ENZ [28] is possible. Ferrell addressed this approach for metallic foils in [29], Berreman - for polar dielectric films in [30]. Nanowire metamaterials support radiative excitations called FB modes. It is should be mentioned, that these FB modes differ from surface plasmon polaritons supported by metal foils. In case of surface plasmon modes, energy propagates along the surfaces of the metal, whereas in FB modes volume charge oscillations are setup across the foil and energy propagates within the bulk of the metal. The bulk polaritons under consideration have transverse wavevectors similar to free space light and exist to the left of the light line. Thus, in Figs. 4 it is interesting to observe the FB modes which usually exist at energies near the ENZ of the hyperbolic metamaterial to the left of the light line [31]. It is worthwhile noting, that in case of spiral nanowire metamaterials, FB modes exist with the absence of ENZ.

From Eqs. 5, 6, 10, 11, the effective permittivity is one important parameter which determines the existence of polaritons in the structure under consideration. Figure 4 depicts a regime map in a $\beta$ - $f$ space, illustrating the regions where FB modes may exist. Fortunately, the frequency range of FB modes existence can be controlled by the wire periodicity $d$, spiral angle $\theta$, diameter of the nanowires $d^{M}$ and distance between the nanowires $S^{M}$. Figure 4 depicts a regime map in a $\beta-f$ space, illustrating the regions where FB polaritons may exist.

The surface polaritons dispersion relations as a function of $d$ is shown in Fig. 4 (a), where $\theta=30^{\circ}, d^{M}=20 \mathrm{~mm}, S^{M}=80 \mathrm{~mm}$. It is clear that FB modes exist at the left region of the light line. The polariton dispersions strongly depend on $d$ parameter, as it can be clearly seen in Fig. 4(a), in which we calculate and plot dispersion relations based on the effective anisotropic permittivity tensor. It should be noted that the frequency range of FB modes existence increases as $d$ increases, i. e. larger periodicity increases the conductivity of the corrugated wire, thereby increasing the effective plasmonic frequency. Furthermore, dramatic tuning of the existence of the polariton spectrum is possible through the modification of the spiral metamaterial structure. The dashed lines are also drawn to facilitate to discussion and to compare the spiral metamaterial properties with the regular nanowire metamaterial properties. Except for $d$, the polariton properties are also dependent on the $\theta$ angle. Here we keep the parameter $d$ of $d=4 \mathrm{~mm}$, and change $\theta$ angle. As observed, the existence region obviously increases to high frequency band with the decrease of $\theta$ angle value. On the whole, the polariton dispersions exhibit an obvious tunability. 


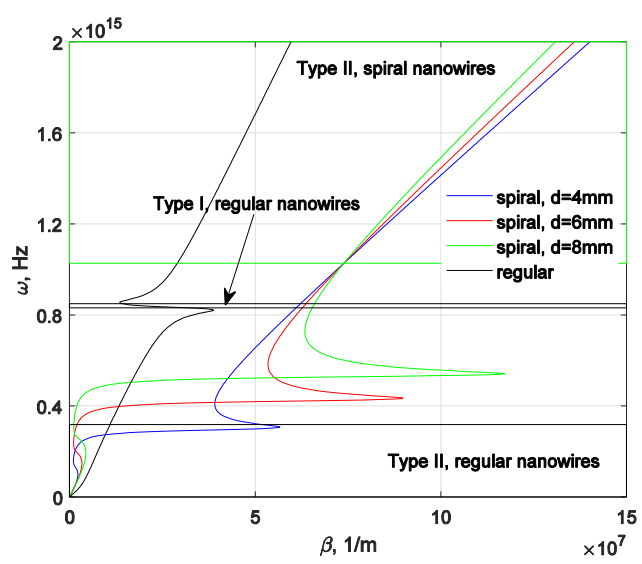

(a)

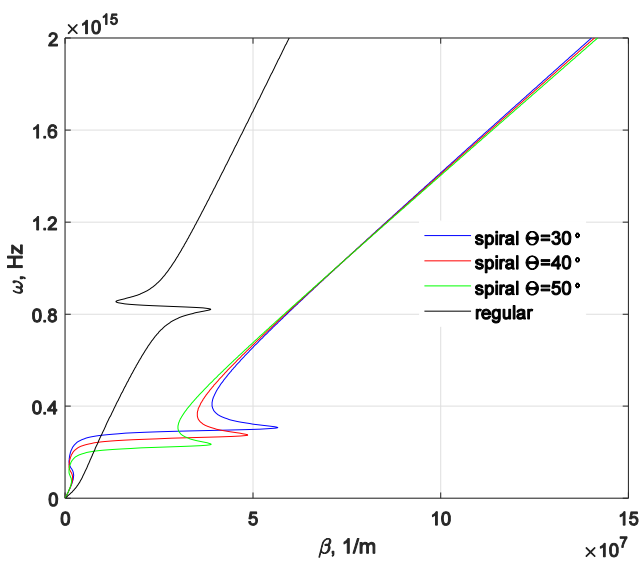

(b)

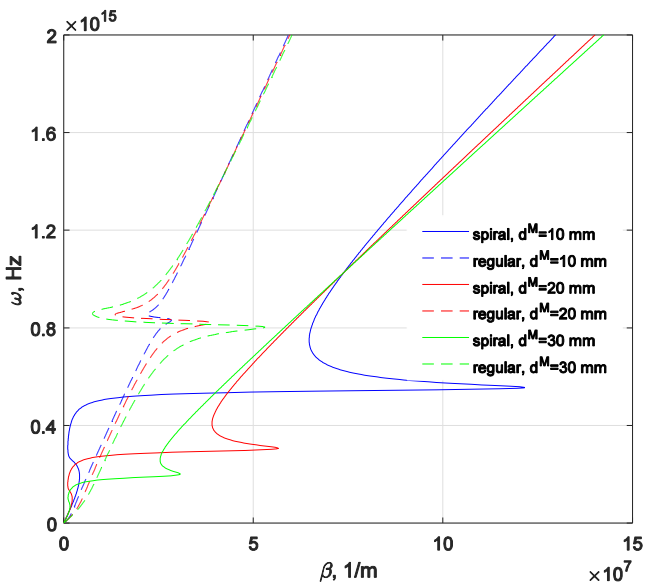

(c) 


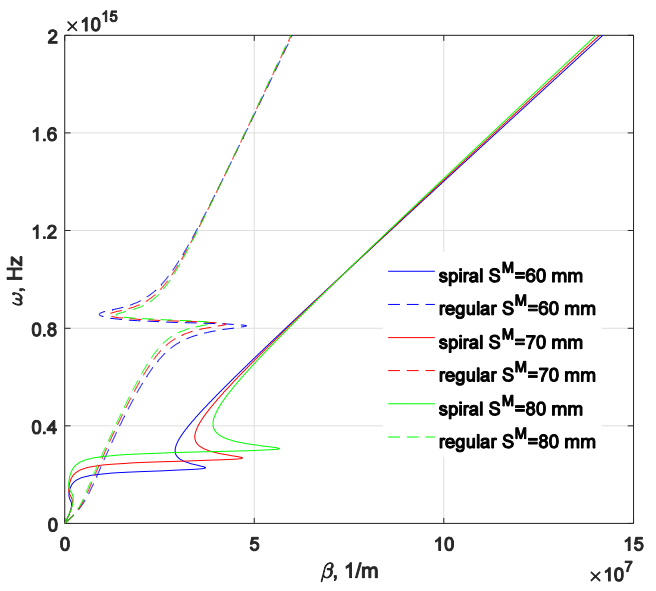

(d)

Fig. 4. The dependences of dispersion relations of surface polaritons on (a) corrugated wire periodicity $d$, (b) spiral angle $\theta$, (c) diameter of the nanowires $d^{M}$, and (d) distance between the nanowires $S^{M}$.

\section{Conclusion}

In conclusion, we have theoretically studied the electromagnetic properties of spiral nanowire metamaterials based on the permittivity homogenization model. Developed theoretical model allows to design and investigate spoof plasmon devices with spiral textures in the optical frequency range. This model allows quantitative study of plasmon polaritons, which can be engineered by the angle of spiral and the number of grooves corrugating the perfectly conducting wire surface. It have been shown that spiral nanowire superlattice based metamaterials exhibit unique micropscopic radiative bulk plasmon resonances called FerrellBerreman modes that can be excited with free-space light. In the metamaterial, effective medium picture the excitation of these modes is captured in the propagation constant, not in the effective dielectric permittivity constants. We observe these modes as anomalous transmission minima, which lie within the transparency window of the metamaterials. These radiative volume polaritonic modes could be exploited in applications such as sensing, imaging and absorption spectroscopy.

\section{Acknowledgement}

This project has received funding from the European Union's Horizon 2020 research and innovation programme under the Marie Sklodowska Curie grant agreement No 713694 and from Engineering and Physical Sciences Research Council (EPSRC) (Grant No. EP/R024898/1). E.U.R. also acknowledges partial support from the Academic Excellence Project 5-100 proposed by Peter the Great St. Petersburg Polytechnic University.

Disclosures. The authors declare no conflicts of interest.

\section{References}

1. J. B. Pendry, "Negative refraction makes a perfect lens," Phys. Rev. Lett. 85, 3966-3969 (2000).

2. W. L. Barnes, A. Dereux, T. W. Ebbesen, "Surface plasmon subwavelength optics," Nature 424, 824-830 (2003).

3. F. J. García-Vidal, J. B. Pendry, "Collective theory for surface enhanced Raman scattering," Phys. Rev. Lett. 77, 1163-1166 (1996). 
4. Y. Luo, A. Aubry, J. B. Pendry, "Electromagnetic contribution to surface-enhanced Raman scattering from rough metal surfaces: a transformation optics approach,” Phys. Rev. B: Condens. Matter Mater. Phys. 83, $155422(2011)$.

5. J. N. Anker, W. P. Hall, O. Lyandres, N. C. Shah, J. Zhao, R. P. Van Duyne, "Biosensing with plasmonic nanosensors," Nat. Mater. 7, 442-453 (2008).

6. J. Li, J. Ye, C. Chen, L. Hermans, N. Verellen, J. Ryken, H. Jans, W. Van Roy, V. V. Moshchalkov, L. Lagae, P. Van Dorpe, "Biosensing using diffractively coupled plasmonic crystals: the figure of merit revisited," Adv. Opt. Mater. 3, 176-181 (2015).

7. V. E. Ferry, J. N. Munday, H. A. Atwater, "Design considerations for plasmonic photovoltaics," Adv. Mater. 22, 4794-4808 (2010).

8. X. Li, X. Ren, F. Xie, Y. Zhang, T. Xu, B. Wei, W. C. H. Choy, "High-performance organic solar cells with broadband absorption enhancement and reliable reproducibility enabled by collective plasmonic effects," Adv. Opt. Mater. 3, 1220-1231 (2015).

9. N. J. Halas, S. Lal, W.-S. Chang, S. Link, P. Nordlander, "Plasmons in strongly coupled metallic nanostructures," Chem. Rev. 111, 3913-3961 (2011).

10. V. Giannini, A. I. Fernández-Domínguez, S. C. Heck, S. A. Maier, "Plasmonic nanoantennas: fundamentals and their use in controlling the radiative properties of nanoemitters," Chem. Rev. 111, 3888-3912 (2011).

11. G. Goubau, "Surface waves and their application to transmission lines," J. Appl. Phys. 21, 1119-1128 (1950).

12. A. F. Harvey, "Periodic and guiding structures at microwave frequencies," IEEE Trans. Microwave Theory Tech. 8, 30-61 (1960).

13. J. B. Pendry, L. Martín-Moreno, F. J. Garcia-Vidal, "Mimicking surface plasmons with structured surfaces," Science 305, 847- 848 (2004).

14. F. J. Garcia-Vidal, L. Martín-Moreno, J. B. Pendry, "Surfaces with holes in them: new plasmonic metamaterials,". J. Opt. A: Pure Appl. Opt. 7, S97 (2005).

15. T. Ioannidis, T. Gric, A.Gorodetsky, A. Trofimov and E. Rafailov, "Enhancing the properties of plasmonic nanowires", Materials Research Express 6, 065014 (2019).

16. T. Gric, A. Gorodetsky, A. Trofimov, E. Rafailov, "Tunable Plasmonic Properties and Absorption Enhancement in Terahertz Photoconductive Antenna Based on Optimized Plasmonic Nanostructures", J. of Infrared Millimeter and Therahertz waves 39(10), 1028-1038 (2018)

17. S. Lepeshov, A. Gorodetsky, A. Krasnok, N. Toropov, T. A. Vartanyan, P. Belov, A. Alu, and E. U. Rafailov, "Boosting the Terahertz Photoconductive Antenna Performance with Optimized Plasmonic Nanostructures", SciRep 8, 6624 (2018).

18. R. Ferrell, "Predicted radiation of plasma oscillations in metal films," Phys. Rev. 111, 1214-1222 (1958).

19. D.W. Berreman, "Infrared absorption at longitudinal optic frequency in cubic crystal films," Phys. Rev. 130, 2193-2198 (1963).

20. P. B. Johnson, R. W. Christy, "Optical constants of the noble metals," Phys. Rev. B 6, 4370 (1972).

21. C. Oubre, P. Nordlander, "Finite-difference time-domain studies of the optical properties of nanoshell dimers," J. Phys. Chem. B 109(20), 10042-10051 (2005).

22. R. Starko-Bowes, J. Atkinson, W. Newman, H. Hu, T. Kallos, G. Palikaras, R. Fedosejevs, S. Pramanik, Z. Jacob, "Optical characterization of Epsilon Near Zero, Epsilon Near Pole and hyperbolic response in nanowire metamaterials," J. Opt. Soc. Am. B 32(10), 2074-2080 (2015).

23. I. Iorsh, A. Orlov, P. Belov, Y. Kivshar, "Interface modes in nanostructured metal-dielectric metamaterials," Appl. Phys. Lett., 99, 151914 (2011).

24. S. Molesky, C. J. Dewalt, and Z. Jacob, "High temperature epsilon-near-zero and epsilon-near-pole metamaterial emitters for thermophotovoltaics," Opt. Express 21(S1), A96-A110 (2013).

25. S. Vassant, J. -P. Hugonin, F. Marquier, and J. -J. Greffet, "Berreman mode and epsilon near zero mode," Opt. Express 20, 23971-23977 (2012).

26. S. Vassant, A. Archambault, F. Marquier, F. Pardo, U. Gennser, A. Cavanna, J. Pelouard, and J.-J. Greffet, "Epsilon-near-zero mode for active optoelectronic devices," Phys. Rev. Lett. 109, 237401 (2012).

27. S. Campione, I. Brener, F. Marquier, "Theory of epsilon-near-zero modes in ultrathin films," Physical Review B 91, 121408 (2015)

28. K. Kliewer, R. Fuchs, "Collective electronic motion in a metallic slab," Phys. Rev. 153, 498-512 (1967).

29. A. McAlister, E. Stern, "Plasma resonance absorption in thin metal films," Phys. Rev. 132, 1599-1602 (1963).

30. D. W. Berreman, "Infrared absorption at longitudinal optic frequency in cubic crystal films," Phys. Rev. 130, 2193-2198 (1963)

31. W. D. Newman, C. L. Cortes, J. Atkinson, S. Pramanik, R. G. DeCorby Z. Jacob, "Ferrell-Berreman modes in plasmonic epsilon-near-zero media," ACS Photonics 2(1), 2-7 (2015). 